\title{
Transvenous Treatment of Carotid Aneurysms Through Transseptal Access
}

\author{
Kirill Orlov ${ }^{1,2}$, Anil Arat ${ }^{3}$, Alexander Osiev ${ }^{4}$, Vadim Berestov ${ }^{1}, K_{\text {Kdret Aytemir }}{ }^{5}$, Mehmet Akif Topcuoglu $^{6}$, \\ Ethem Murat Arsava ${ }^{6}$
}

\author{
Key words \\ - Cerebral aneurysm \\ - Dissecting aneurysm \\ - Endovascular treatment \\ - Flow diverter \\ - Transseptal access \\ - Transvenous approach
}

\section{Abbreviations and Acronyms \\ CTA: Computed tomography angiography \\ TP: Transseptal puncture}

TSA: Transseptal access

From the ${ }^{\mathbf{1}}$ E.N. Meshalkin Siberian Federal Biomedical Research Center, Novosibirsk, Russian Federation;

${ }^{2}$ Novosibirsk State Medical University, Novosibirsk, Russian Federation; ${ }^{\mathbf{3}}$ Department of Radiology, Hacettepe University Hospitals, Ankara, Turkey; ${ }^{4}$ Moscow Regional Research and Clinical Institute ("MONIKI"), Moscow, Russian Federation;

${ }^{5}$ Department of Cardiology, Hacettepe University Hospitals, HUTF Kardiyoloji ABD, Ankara, Turkey; and ${ }^{\boldsymbol{6}}$ Department of Neurology, Hacettepe University Hospitals, HUTF Noroloji ABD, Ankara, Turkey

To whom correspondence should be addressed: Anil Arat, M.D.

[E-mail: anilarat@hotmail.com]

\section{Supplementary digital content available online.} Citation: World Neurosurg. (2019) 124:459-463.

https://doi.org/10.1016/j.wneu.2018.12.207

Journal homepage: www.journals.elsevier.com/worldneurosurgery

Available online: www.sciencedirect.com

1878-8750/\$ - see front matter (c) 2019 Elsevier Inc. All rights reserved.

\section{BACKGROUND}

Despite the controversy surrounding the endovascular treatment of cervical carotid artery dissections associated with an aneurysm, there is a subgroup of patients in whom clinical decision favors endovascular treatment based on the very low (as low as $0 \%$ morbidity in large clinical series) treatment risk. ${ }^{\mathrm{I}}$ Among these lesions, untreated traumatic dissecting aneurysms represent a more specific subset with unique morphologic features ${ }^{2}$ and a worse prognosis ${ }^{3}$ as compared with spontaneous dissections. Therefore, traumatic dissections and aneurysms require a more aggressive treatment strategy. ${ }^{4}$ We report 2 patients, one who was involved in a motor vehicle accident

BACKGROUND: Transseptal puncture has been widely used by cardiologists to reach the left side of the heart through a transvenous access. Rarely, it also can be used to pass into the supra-aortic arteries from the venous side when conventional transarterial access pathways (transfemoral, transradial/brachial routes, or direct carotid puncture) are likely to fail.

CASE DESCRIPTION: We report 2 cases of transvenous femoral access followed by transseptal access to aorta to treat dissecting carotid artery aneurysms at the level of the skull base with flow diverters. In one case, multiple cervical arterial bypass operations and in the other a rare anomaly of the aortic arch precluded endovascular treatment through conventional routes.

CONCLUSIONS: Transvenous-transseptal access enabled treatment of both cases easily and without complications. On follow-up computed tomography angiograms, both flow diverters were patent, there were no residual aneurysms, and no neurologic or cardiac adverse events in either patient.

and another with Eagle syndrome, a condition in which repetitive carotid trauma due to an elongated styloid process is responsible for carotid dissecting aneurysms. ${ }^{5}$ In both cases, previously reported routes of access to the common carotid artery were not feasible, and a transvenous-transseptal approach was successfully performed to treat dissecting carotid aneurysms.

\section{CASE DESCRIPTIONS}

Both of the patients provided consent for this publication in addition to their previous consent for their treatment.

\section{CASE 1}

A young man suffered a severe motor vehicle accident resulting in a traumatic dissection of the distal aortic arch and an associated large pseudoaneurysm. He underwent an urgent left subclavian-left common carotid bypass followed by carotid-carotid bypass from the right common carotid artery to the contralateral common carotid artery, and finally the dissected aortic segment was treated by placement of an aortic graft. Just after the final treatment, he developed an aortoiliac thrombosis and underwent aortofemoral bypass surgery. One month after the last surgery, while under dual antiplatelet treatment, he had transient right-sided hemiparesis. Computed tomography angiography (CTA) of the head and neck revealed patency of the bypasses and a partially thrombosed dissecting left-sided, high cervical carotid aneurysm (Figure 1A).

Because of the anticipated difficulties and risks of accessing the aortofemoral bypasses in the subacute period as well as navigating catheters into the angled left common carotid artery ostium, which was partially covered by the aortic graft, a femoral route for endovascular treatment was not attempted. A left brachial access and navigation through the bypass was also considered to be risky, with a questionable chance of success due to the angulation and stenosis at the suture line of the bypass. Direct percutaneous carotid access in the recently operated neck also was not considered.

Finally, under dual antiplatelet therapy (clopidogrel $75 \mathrm{mg} /$ day, aspirin $75 \mathrm{mg} /$ day) and general anesthesia, a right femoral venous access was established and the right atrium was catheterized with an 8F Destination sheath (Terumo Medical Corp., 

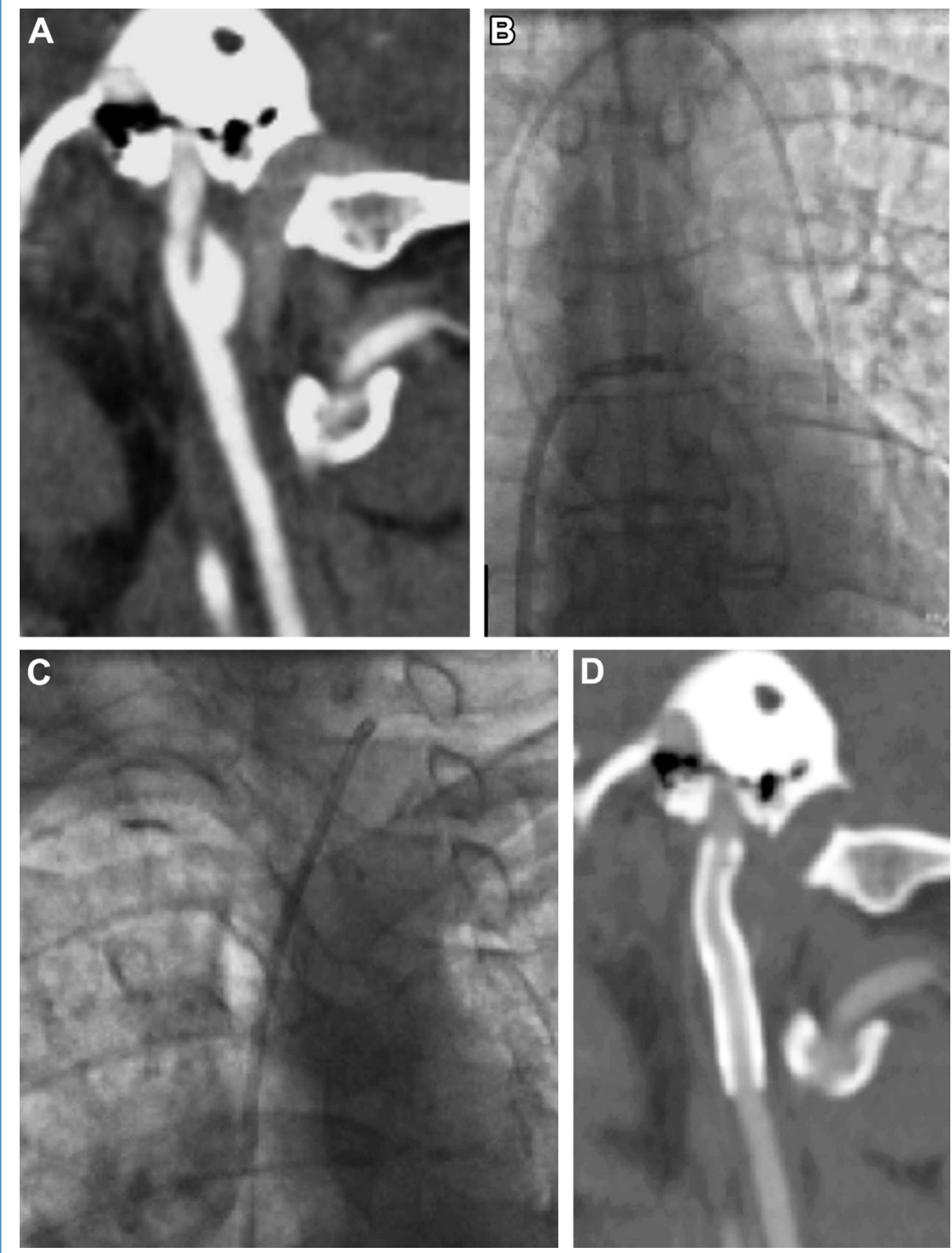

Figure 1. (A) Computed tomography angiogram of the head demonstrates a traumatic aneurysm of the left internal carotid artery. (B) Access to the aorta with the guidewire was achieved via

transseptal puncture and then navigation of the guidewire through the left atrium, mitral valve, left ventricle, and finally aortic valve. The distal access catheter was navigated into the aorta through the transseptal sheath and over the guidewire. (C) Then, the distal access catheter was pulled back and the origin of the left common carotid artery was engaged and this artery was catheterized. The figure shows the tip of the distal access catheter in the proximal left common carotid artery. (D) Control computed tomography angiogram at 6 months shows the patency of the carotid artery and no evidence of a residual aneurysm.

Elkton, Maryland, USA). Transseptal access (TSA) was obtained with a Brockenbrough needle (Medtronic CardioVascular, Munds View, Minnesota, USA) under transesophageal ultrasound guidance. The needle was replaced with a $6 \mathrm{~F}$ guiding catheter that was used to catheterize the left common carotid artery after traversing the mitral and aortic valves (Figure 1B, C). The aneurysm was treated by deployment of a $5 \times 30-\mathrm{mm}$ Pipeline device (Medtronic, Irvine, California, USA) delivered through a Marksman microcatheter (Medtronic) microcatheter. The patient tolerated the procedure well. On follow-up, the patient remained asymptomatic and control computed tomography studies on postoperative day 3 and at 6 months revealed complete thrombosis of the aneurysm (Figure 1D) and patency of the carotid artery. The patient was doing well 72 months after treatment.

\section{CASE 2}

A young woman presented with acute exacerbation of her chronic, right-sided head and neck pain, which had recently ceased to respond to oral analgesics. Magnetic resonance angiography of the head revealed a thrombosed cervical internal carotid artery aneurysm and narrowing of the right distal internal carotid artery. The patient was diagnosed with acute dissection of the internal carotid artery and was started on oral anticoagulation. Soon after discharge, she presented with worsening headaches. CTA revealed recanalization of the dissecting aneurysm and an elongated (approximately $5 \mathrm{~cm}$ ) right styloid process which showed a notch adjacent to the dissecting aneurysm (Supplementary Figure 1A). Both the CTA and a magnetic resonance angiogram showed a right-sided aortic arch and a small $(4 \mathrm{~mm}$ in diameter) right common carotid artery that directly arose from the aortic arch as its second branch and ascended deep in the neck adjacent to the vertebral column (Figure 2A and Supplementary Figure 1B). A right carotid angiogram was extremely hard to perform due to the multiple angulations of the aortic arch and due to the steep takeoff of the right common carotid artery (Supplementary Figure 1C, Figure 2B) as the first supra-aortic artery. The angiogram showed a dissecting aneurysm of the distal right internal carotid artery (Figure 2C). The senior interventionalist was able to obtain a selective angiogram of the right internal carotid artery only with the use of a microcatheter placed through the Simmons 2-type diagnostic catheter. The patient refused to have surgical treatment for Eagle syndrome. A suggestion to treat the aneurysm was made based on the unstable nature of the aneurysm, possibility of further carotid injury as part of Eagle syndrome, and the young age of the patient.

Because navigation of a triaxial catheter system to the right internal carotid artery would be at least as difficult through a 

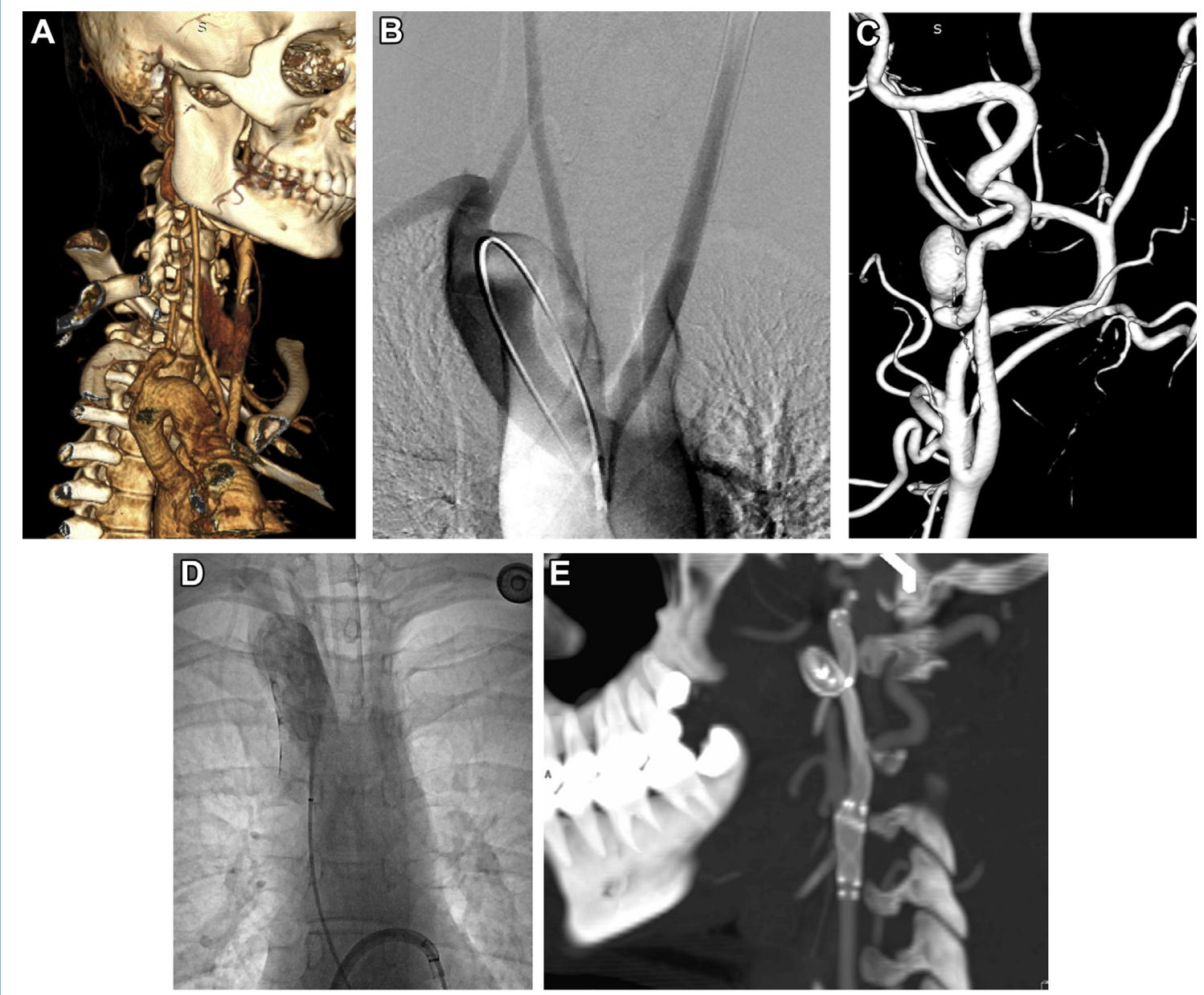

Figure 2. (A) Volume-rendered image of the computed tomography angiogram of the head and neck reveals the anomalous aortic arch and the supra-aortic major arteries. The first branch is the left common carotid artery, the right common carotid artery (CCA) originates directly originates from the arch, as its second branch, with an acute angle and it is hypoplastic. It ascends alongside the right vertebral artery deep in the neck. The right subclavian artery also arises directly from the aortic arch as the third branch. (B) Aortogram obtained with a

Simmons-2 type catheter shows that the anomalous aortic arch has a steep angulation as it descends from the right side to the left. The left subclavian artery arises from the aorta on the left side with an infundibulum at its origin. The left CCA is slightly

smaller in diameter as compared to the ipsilateral cervical vertebral artery. (C) The dissecting aneurysm of the right interna carotid artery (ICA) as seen on 3-dimensional views obtained from the rotational angiogram. (D) The root of the aorta is accessed with a Navien 058 catheter (Medtronic) which was navigated through an $8.5 \mathrm{~F}$ sheath. This sheath was navigated to the right atrium from the venous side, then its tip was placed into the left atrium after a transseptal puncture. The Navien catheter made a loop in the heart by going through, in order, the mitral valve, the left ventricle, and the aortic valve to reach the root of the aorta. (E) Computed tomography angiogram obtained at 8 months shows a patent right ICA and no evidence of a residual aneurysm.

brachial approach as the femoral access and because the small and anomalous carotid artery lied deep in the neck (by the vertebral column, as noted in Figure 2A and Supplementary Figure 1B), TSA was considered to be the best approach for endovascular treatment. The patient was administered clopidogrel for 7 days. Under general anesthesia and through a femoral venous access, an $8.5 \mathrm{~F}$ steerable introducer sheath (Agilis NXT; St. Jude Medical, St. Paul, Minnesota, USA) was placed in the right atrium and under transesophageal ultrasound guidance, the atrial septum was pierced with a transseptal needle (BRK; St. Jude Medical) (Video 1).
The catheter was steered by its handle and kept at the TSA site by the first operator while the second operator first navigated a Neuron Max catheter (Penumbra, Alameda, California, USA), which ended up being too short to reach the aorta. Then, a 5 F $125-\mathrm{cm}$ Navien distal access catheter (Medtronic) was exchanged with the Neuron Max catheter. The Navien catheter was navigated through the mitral and aortic valves to 
reach the root of the aorta and finally the right common carotid artery (Figure 2D). Because this artery originated from the anomalous aortic arch at a straight trajectory in relevance to the outflow tract of the left ventricle (Supplementary Figure 2), catheterization of the right common carotid artery was straightforward. Once the right common carotid artery was accessed and angiograms were performed (Supplementary Figure 3A), a Headway 027 catheter (Microvention, Tustin, California, USA) was used to deploy a $6 \times 50-\mathrm{mm}$ Derivo device (Acandis, Forsheim, Germany) (Supplementary Figure 3B). The proximal part of the stent was pinned by deployment of a $4.5 \times 30-\mathrm{mm}$ Neuroform stent (Stryker, Kalamazoo, Michigan, USA) whereas the distal part was apposed with a $4.5 \times 2$ I$\mathrm{mm}$ Neuroform Atlas stent (Stryker) placed through an SL-Io microcatheter (Stryker). There were no complications. At 3 months, an angiogram was obtained and showed partial opacification of the aneurysm (Supplementary Figure 3C), a cardiac echocardiogram was unremarkable. At 8 months, the patient reported no symptoms, the aneurysm was totally occluded on CTA (Figure 2E) and the carotid artery was patent. She was doing well at a recent I-year clinical follow-up.

\section{CONCLUSIONS}

It is suggested that traumatic pseudoaneurysm formation is one of the indications for endovascular treatment of dissecting carotid aneurysms. ${ }^{6}$ The trauma may be a major blunt impact, as in our first case. It may also be secondary to subtle but repetitive injury, such as the rare case of the impingement of the carotid artery by an anomalous styloid process during forced movements of the neck, as seen in our second case. The latter form also has been referred to as a "self-stabbing phenomenon." A review by Spanos et al. ${ }^{8}$ shows that the endovascular treatment of traumatic dissections is associated with a very low risk of serious adverse events. As a matter of fact, a detailed analysis of this recent publication shows that the rate of serious adverse events for endovascular treatment of these aneurysms is zero percent in those series reported after the year 20I0. Hence, endovascular treatment of traumatic cervical carotid artery aneurysms appears to be a reasonable management approach, especially for traumatic aneurysms which, as in our cases, appear saccular and are more prone to enlarge. ${ }^{9}$

Currently transfemoral access to the carotid artery is the standard approach for endovascular treatment of vascular lesions of the anterior circulation. A meta-analysis by Das et al. ${ }^{\text {IO }}$ showed that transfemoral approach is associated with $3 \%-\mathrm{II} \%$ of access-related complications, approximately I $\%$ of which are serious access-site complications (pseudoaneurysm formation, retroperitoneal hematoma, or distal ischemia) regardless of the use of a closure device. Alternative access through radial, brachial, or cervical routes may be necessary in patients with tortuosity of supra-aortic arteries, ${ }^{\mathrm{II}, \mathrm{I2}}$ especially when flow diverters are used, ${ }^{\mathrm{I} 3}$ because $\mathrm{I} \%-6 \%$ of neuroendovascular procedures fail secondary to access-related problems. ${ }^{\text {II }} \mathrm{A}$ large-scale randomized study on safety of radial versus femoral access showed that the bleeding complications were approximately $\mathrm{I} \%$ and similar in both types of access. ${ }^{\text {I4 }}$ In contrast, the complication rate of cervical access for neurovascular procedures is approximately $7 \% .{ }^{\text {IO,I2 }}$ The life-threatening risks of cervical hematoma or dissection ${ }^{12,15}$ in direct access are not eliminated by arterial closure devices ${ }^{\text {II }}$ and at times, may be precipitated by them. ${ }^{\text {I2 }}$

In contrast, the risk of transseptal puncture (TP)/TSA is low, in the range of $0.5 \%-2 \%$. ${ }^{\mathrm{I}} \mathrm{It}$ is a conventional technique that dates back to $1959^{17}$ and is currently used by cardiologists widely for ablation of atrial fibrillation. A recently published series of 504 consecutive TPs reports no access-related complications using contemporary access methods. ${ }^{18}$ Likewise, a randomized study comparing 2 different TP methods found that the complication rate with both methods was zero percent. ${ }^{16}$ For the endovascular treatment of valvular disease, it is not unusual to perform an angioplasty of the TP site with large balloons (up to $15 \mathrm{~mm}$ in diameter) without complications secondary to the TSA.

In our patients, our inability to secure internal carotid artery access led to the consideration of alternative access pathways. Among these, the radial or brachial accesses would be futile in patient 2 due to the angulations between the right subclavian artery and the anomalous aorta as well as the right common carotid artery and the aorta. In this patient, direct access to the common carotid artery would carry a high risk because the artery was small and lied deep in the neck, along the vertebral artery due to its aberrant course. The brachial and percutaneous accesses in the first patient would be risky due to multiple operations, including bypasses, of the subclavian arteries and the left common carotid artery, which would not only make access risky but also make navigation difficult due to the significantly altered arterial anatomy. Direct surgical exposure for a neuroendovascular procedure would either be unviable or would carry at least $10 \%$ risk of carotid damage ${ }^{\text {I9 }}$ borne on the reasons stated previously. In both cases, TP had a well-defined and very small risk and also predictable consequences. In both cases, once the TP was performed, aortic and carotid access was straightforward.

After TSA is secured, going through the mitral and aortic valves in the direction of the blood flow is technically easy with negligible risk. Performed for the first time in 1988 to treat aortic coarctation in an infant, ${ }^{17}$ aortic access after septal puncture has been performed for a variety of treatments. Carotid stenting via this route was reported more than 20 years $\mathrm{ago}^{20}$ and has been occasionally used in cases with supra-aortic tortuosity ${ }^{2 I}$ without any permanent adverse events related to such access being reported so far.

Transvenous/TSA for cerebral aneurysm treatment has not been previously reported. Our cases were performed safely and relatively easily by a team of experienced neuroendovascular specialists and cardiologists. Yet, there are potential complications: reversible arrhythmia (cardiac stimulation by the guiding catheter) and damage to the mitral valve is possible in aortic access after TSA. $^{17}$ These are unlikely to occur with the small-bore soft distal access catheters we use in aneurysm treatment. Another drawback is the kickback of the transseptal sheath, and this is why we suggest that the whole procedure should be performed together with cardiologists experienced in cardiac 
interventions. Other drawbacks we came across were related to the "intracardiac loop" of the distal access catheter, as noted in Figures 1B and 2D. This loop gets larger as the resistance to microcatheter navigation increases at the level of the carotid siphon. Longer distal access catheters also are needed because some of the length of the access catheter is used up by this loop. Despite these drawbacks, we have shown that transvenous treatment of cerebral arterial aneurysms via TSA appears to be feasible in those rare cases in which arterial access is risky or not possible.

\section{REFERENCES}

I. Seth R, Obuchowski AM, Zoarski GH. Endovascular repair of traumatic cervical internal carotid artery injuries: a safe and effective treatment option. AJNR Am J Neuroradiol. 2013;34:I2I9-I226.

2. Foreman PM, Harrigan MR. Blunt traumatic extracranial cerebrovascular injury and ischemic stroke. Cerebrovasc Dis Extra. 2017;7:72-83.

3. Brzezicki G, Rivet DJ, Reavey-Cantwell J. Pipeline Embolization Device for treatment of high cervical and skull base carotid artery dissections: clinical case series. J Neurointerv Surg. 2016;8:722-728.

4. Galyfos G, Filis K, Sigala F, Sianou A. Traumatic carotid artery dissection: a different entity without specific guidelines. Vasc Specialist Int. 20I6;32:I-5.

5. Todo T, Alexander M, Stokol C, et al. Eagle syndrome revisited: cerebrovascular complications. Ann Vasc Surg. 20I2;26:729. eI-e5.

6. Xianjun $\mathrm{H}$, Zhiming Z. A systematic review of endovascular management of internal carotid artery dissections. Interv Neurol. 2013;I:I64-I70.
7. Razak A, Short JL, Hussain SI. Carotid artery dissection due to elongated styloid process: a selfstabbing phenomenon. J Neuroimaging. 2014;24: 298-30I.

8. Spanos K, Karathanos C, Stamoulis K, et al. Endovascular treatment of traumatic internal carotid artery pseudoaneurysm. Injury. 2016;47: 307-312.

9. Foreman PM, Griessenauer CJ, Falola M, et al. Extracranial traumatic aneurysms due to blunt cerebrovascular injury. J Neurosurg. 2014;120: I437-I445.

Io. Das R, Ahmed K, Athanasiou T, et al. Arterial closure devices versus manual compression for femoral haemostasis in interventional radiological procedures: a systematic review and meta-analysis. Cardiovasc Intervent Radiol. 20II;34:723-738.

II. Dorfer C, Standhardt H, Gruber A, et al. Direct percutaneous puncture approach versus surgical cutdown technique for intracranial neuroendovascular procedures: technical aspects. World Neurosurg. 2012;77:192-200.

I2. Iosif C, Clarençon F, Di Maria F, et al. Combined Angio-Seal ${ }^{\mathrm{TM}}$ and stenting rescue treatment in a case of iatrogenic common carotid artery dissection during direct puncture for ruptured intracranial aneurysm embolization: a technical note. J Neuroradiol. 2013;40:130-I33.

I3. Peitz GW, Kura B, Johnson JN, et al. Transradial approach for deployment of a flow diverter for an intracranial aneurysm in a patient with a type-3 aortic arch. J Vasc Interv Neurol. 20I7;9:42-44.

I4. Jolly SS, Yusuf S, Cairns J, et al. Radial versus femoral access for coronary angiography and intervention in patients with acute coronary syndromes (RIVAL): a randomised, parallel group, multicentre trial. Lancet. 20II;377:I409-I420.

I5. Ankay Yilbas A, Kanburoglu C, Uzumcugil F, et al. Progressive hematoma in anterior neck after endovascular treatment of middle cerebral artery aneurysm. Rev Bras Anestesiol. 2018;68:194-Ig6 [in Portuguese].

I6. Kataria V, Berte B, Vandekerckhove Y, et al. Modified transseptal puncture technique in challenging septa: a randomized comparison to conventional technique. Biomed Res Int. 20I7;2017: 2351925.

I7. Joseph G. Commentary: transcardiac access to the ascending aorta and beyond. J Endovasc Ther. 2015; 22:385-387.

I8. Lehrmann H, Schneider J, Jadidi AS, et al Transseptal access for left atrial ablation: the catheter-probing techniques are not without risk. J Cardiovasc Electrophysiol. 2014;25:479-484.

I9. Shin DS, Yilmaz A, Ozkul A, et al. Direct carotic exposure for neuroendovascular approaches. J Neurol Surg A Cent Eur Neurosurg. 2016;77:505-510.

20. Joseph G, Krishnaswami S, Baruah DK, et al Transseptal approach to aortography and carotid artery stenting in pulseless disease. Cathet Cardiovasc Diagn. I997;40:4I6-420.

2I. Yoon YS, Shim WH. Transseptal approach for stent implantation in rightinternal carotid artery stenosis. J Invasive Cardiol. 2000;12:70-74.

Conflict of interest statement: The authors declare that the article content was composed in the absence of any commercial or financial relationships that could be construed as a potential conflict of interest.

Received 6 November 2018; accepted 26 December 2018

Citation: World Neurosurg. (2019) 124:459-463. https://doi.org/10.1016/j.wneu.2018.12.207

Journal homepage: www.journals.elsevier.com/worldneurosurgery

Available online: www.sciencedirect.com

1878-8750/\$ - see front matter (c) 2019 Elsevier Inc. All rights reserved. 


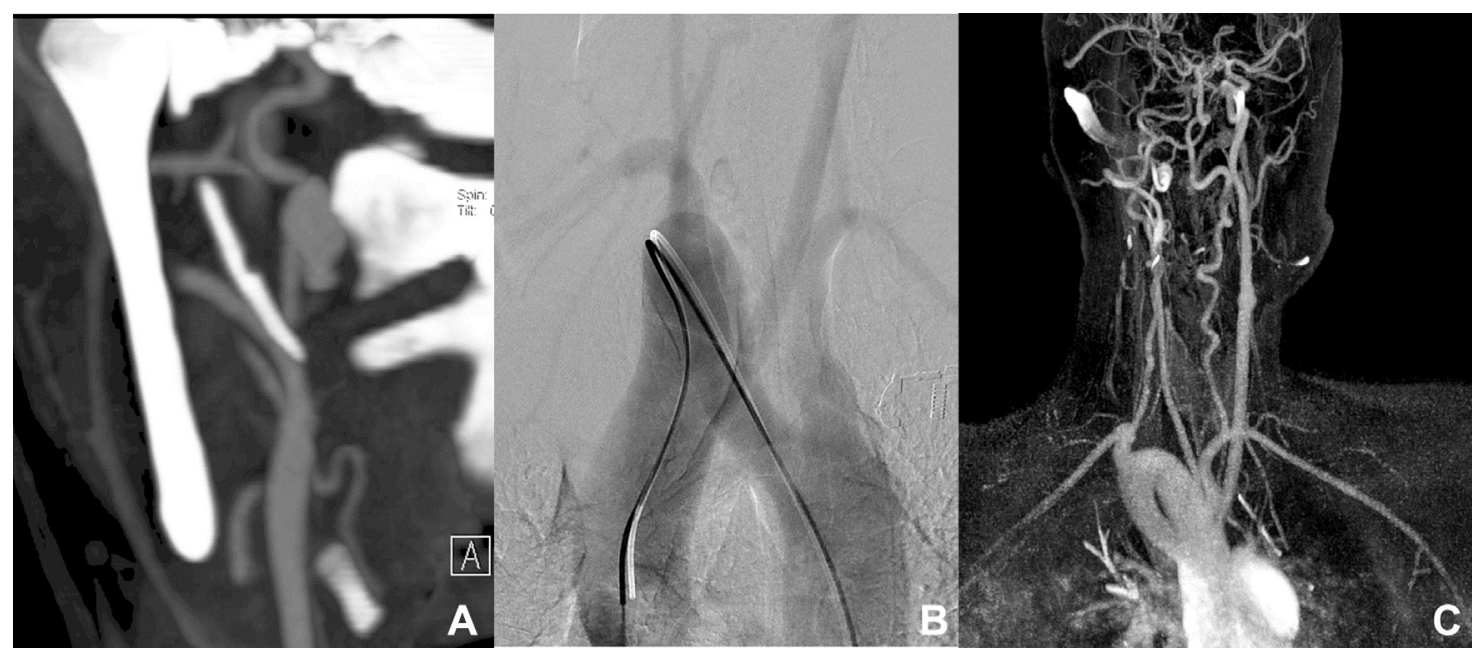

Supplementary Figure 1. (A) An elongated styloid process is seen on the maximum intensity projection images obtained from the computed tomography angiogram, of note is the "notch" on the styloid process adjacent to the dissecting aneurysm. (B) Contrast-enhanced magnetic resonance angiography of the

head and neck demonstrates the small size of the right common carotid artery and its deep trajectory in the neck. (C) The origins of both common carotid arteries, immediately adjacent to the aortic valve noted on the aortogram obtained with a high frame rate.

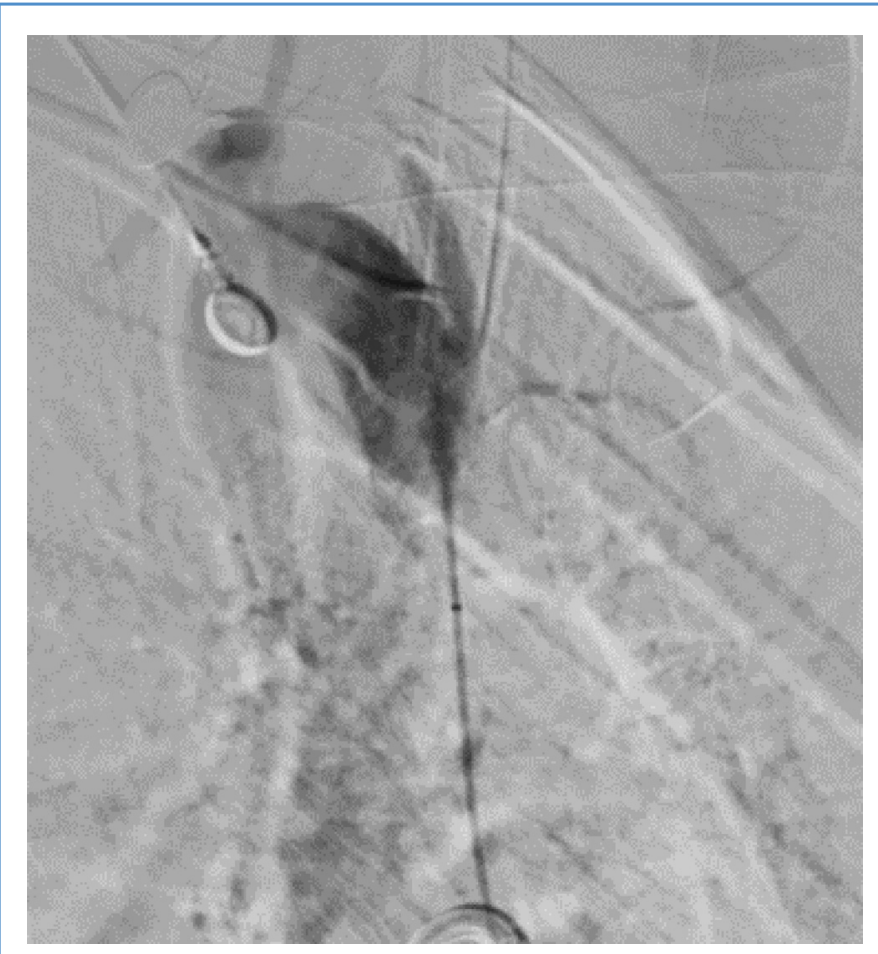

Supplementary Figure 2. Angiogram obtained through the Navien catheter after transseptal navigation into the arterial side reveals that the origin of the right common carotid artery is at a straight trajectory when transcardiac navigation is used. 


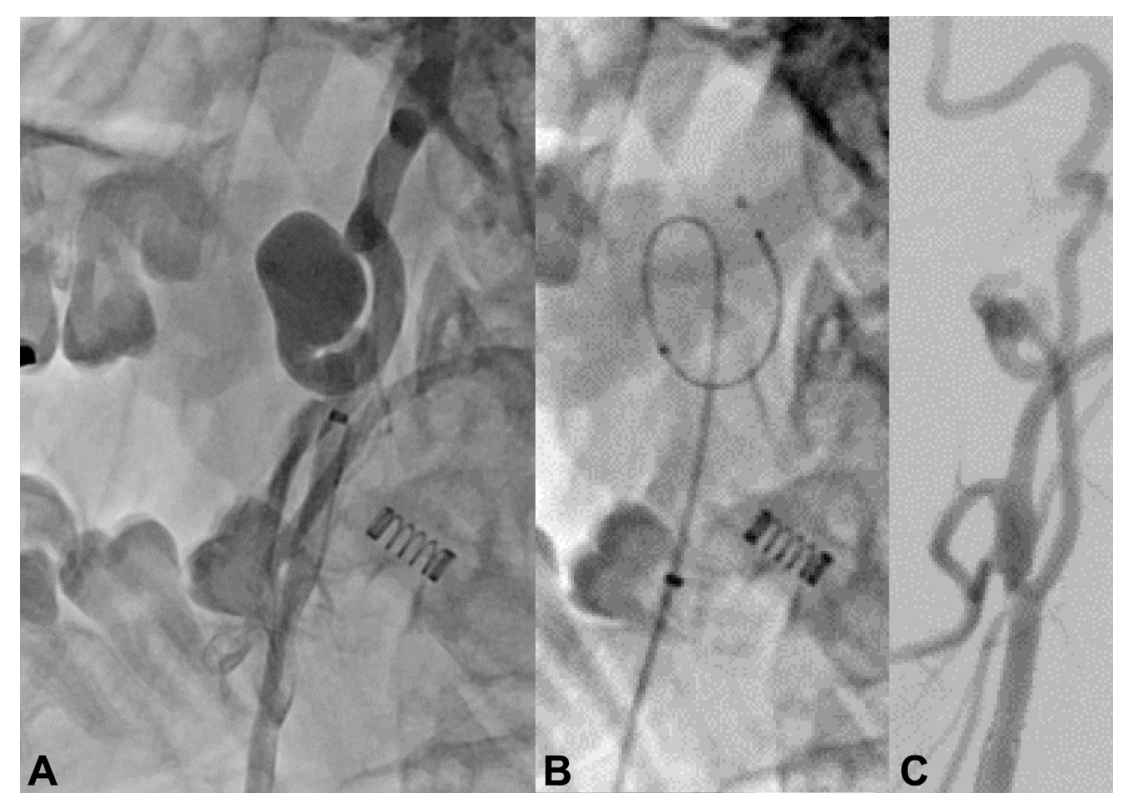

Supplementary Figure 3. (A) Angiogram obtained through the distal access catheter reveals the internal carotid aneurysm. (B) The aneurysm is bypassed with a microcatheter and Derivo device (Acandis) is being deployed. (C) A control angiogram at 3 months shows reduced contrast filling in the aneurysm and patency of the carotid artery without significant neointimal hyperplasia. 